\title{
Atypical Perfusion Manifestation in Migraine with Aura
}

\author{
Jacobo Lester ${ }^{a}$ Jesús Carlos Bustamante ${ }^{b} \quad$ Carla García-Moreno $^{c}$ \\ Enrique Klériga ${ }^{b}$ \\ aNeurology, Instituto Mexicano de Neurociencias, Huixquilucan, Mexico; b Neurosurgery, \\ Instituto Mexicano de Neurociencias, Huixquilucan, Mexico; 'Magnetic Resonance, Hospital \\ Angeles Lomas, Huixquilucan, Mexico
}

\section{Keywords}

Migraine $\cdot$ Aura $\cdot$ Perfusion

\begin{abstract}
Migraine with aura may be confused with a stroke. Magnetic resonance imaging is an important tool for the differential diagnosis. Cerebral hypoperfusion has been described in classic migraine, mainly during the aura. A 47-year-old male had an unremarkable past medical history. After sneezing, he developed a left hemi hypoesthesia, bitemporal vision loss, photopsia, and some distortion in the position of letters and words. This lasted $<1 \mathrm{~h}$, and it was followed by a severe headache. A magnetic resonance angiography was performed during the headache. It showed a left hemispheric hypoperfusion that did not correlate with the symptoms described by the patient. It is believed that during the aura, cerebral blood flow decreases, leading to hypoxia and decreased cellular energy generation, and these metabolic alterations define the symptoms of the patient. In our case, we documented brain hypoperfusion during the headache in the ipsilateral brain hemisphere to the symptoms, which has no clinical correlation. This condition could be due to spasm in the capillary arteries, and it may persist and influence the clinical manifestations during the headache phase in migraine with aura. A state of generalized cerebral hyperperfusion has been suggested, and there may be a coexistence of both phenomena for some period. This may open a new line of research regarding the pathophysiology and vascular changes of migraine with aura.
\end{abstract}

\section{Karger ${ }^{\prime \prime}=$}




\section{Introduction}

During migraine aura, it is considered there is a decreased cortical propagation of neuronal discharges followed by full recovery of cortical electrical activity. It is accompanied by minutes of hypoperfusion followed by mild hyperperfusion causing this last, headache phase [1].

Visual complaints, sensory, aphasic symptoms, and hemiparesis may develop prior to the onset of headache in migraine with aura (MA). These symptoms last from 5 to $60 \mathrm{~min}$, and at least one of these symptoms must be unilateral, reversible, and followed by headache [2].

MA can be associated with some changes in cerebral perfusion. These changes have also been described in other conditions that may be confused, mainly with a stroke. In those cases, magnetic resonance imaging (MRI) may be helpful with the differential diagnosis, considering that in very few occasions, migraine may be the cause of a stroke, due to severe arterial constriction [1-3]. We present an uncommon case of a patient with MA with prolonged and atypical changes in brain perfusion [1] after resolution of the aura and during the headache phase.

\section{Case Report}

This is the case of a 47-year-old right-handed man, without a family history of migraine. Early morning on May 7, 2020, after going to the restroom, he sneezed twice and complained of decreased sensitivity in the left side of the body, predominantly in the arm, visual loss in the bilateral temporal field, and he also saw bright lights, which lasted less than an hour. Those symptoms disappeared and then began with bifrontal oppressive headache, 8-9/10 on the visual analog scale (VAS), along with photophobia, phonophobia, dizziness, nausea, and no vomiting, and he changed letters or words order. Therefore, he was transferred to the emergency room.

Previously in October 2018 and 2 weeks prior to his symptoms, after going to the restroom, he presented similar symptomatology which was also associated with sneezing twice. Later on, he manifested paresthesia of the left side of the body, numbness in the left half of the tongue, and saw bright colors, which disappeared after a 30-min period. It was followed by headache with the characteristics mentioned above that also disappeared after an hour. He had an MRI that was reported with normal perfusion, although he no longer had the images.

Upon arrival at the emergency room, the patient had dysarthria and word order alteration. The rest of the neurological examination was within normal limits. Clinical signs decreased partially with ketoprofen $100 \mathrm{mg}$ and metoclopramide $10 \mathrm{mg}$, a single dose. The pain persisted with a 6/10 score on VAS, and then dexamethasone $8 \mathrm{mg}$ was administered intravenously, which led to total pain relief.

A magnetic resonance angiography (MRA) was performed during headache, and it showed asymmetric bilateral subcortical microangiopathic images of nonspecific origin, diffusion, and normal angiographic phase. However, a delay in the mean transit time was revealed in the left hemisphere and in the right temporal lobe on a smaller scale, which may be associated with an exacerbated migraine condition (Fig. 1).

The patient had a normal electroencephalogram. We also ruled out a transient cerebral ischemia. At 1 year of follow-up, the patient remains free of new episodes of migraine and aura.

\section{Discussion}

Changes in cerebral perfusion on MRI are sometimes difficult to document in MA since they usually occur during the aura or the initial phase of the headache. Brain hypoperfusion is more frequent on the posterior circulation in $71.4 \%$ of the cases. In $28.6 \%$, it occurs in the

\section{Karger'}




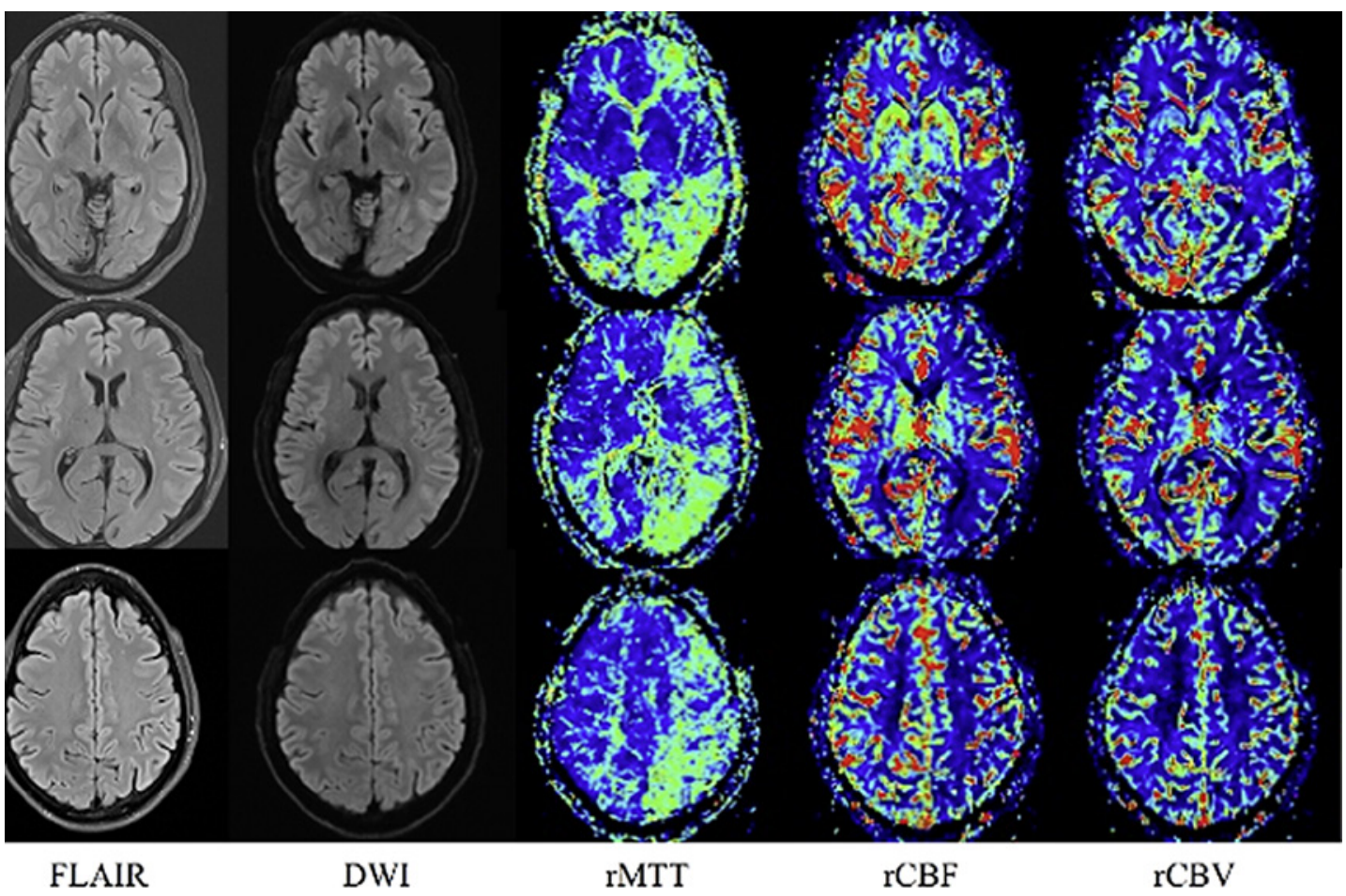

Fig. 1. Magnetic resonance imaging. Software: Siemens Skyra Sit, Version E11. Normal axial FLAIR and axial DWI ( $b=1,000 \mathrm{~s} / \mathrm{mm}^{2}$ ) without ischemic injury or edema. The cerebral perfusion maps show delay in contrast arrival in the left hemisphere with a prolonged $\mathrm{rMTT}, \mathrm{rCBF}$, and $\mathrm{rCBV}$ in all contiguous slices through the left hemisphere. FLAIR, fluid-attenuated inversion recovery; DWI, diffusion-weighted imaging; rMTT, mean transit time; rCBF, reduced cerebral flow; rCBV, reduced cerebral volume.

rest of the vascular territories [2], and they can be observed generally during the aura [4] and in fewer cases during the progression of the headache. The range of time goes from $11 \mathrm{~h}$ [5] up to 12 days, but in all cases, the changes were reversible [6]. In these latter circumstances, hypoperfusion is regional [2]. It is considered that during the aura, cerebral blood flow decreases, with the consequent ischemia, tissue hypoxia, and cellular metabolic alterations in the production of adenosine triphosphate, as well as a decrease in tissue $\mathrm{pH}$ in those areas where the blood flow is altered. This usually matches with the patient's clinical manifestations [6], in contrast to our patient, who presented these changes in perfusion with the aura solved and during a longer phase with headache.

Our patient had visual, language, and left body components during the aura and subsequently presented the headache phase, which constitutes an infrequent finding. When we performed the brain MRA $6 \mathrm{~h}$ after the onset of clinical manifestations, we found extensive left hemispheric hypoperfusion, that is, in the opposite side of the brain relevant to the patient's clinical manifestations.

It is of notice that the MA in our patient was triggered with sneezing. It has been proposed that this is possible in that it is due to activation of the trigeminal system and that it could also be associated with some visual symptoms in migraine [7]. Likewise, in relation to the cranial autonomic symptoms, it may activate central mechanisms, mainly in the hypothalamus [8], although other causes should also be ruled out, such as Chiari malformations, as we did in our patient [9].

It is of interest in this case, during the aura phase, the presence of a bitemporal visual deficit. This could be associated with the activation of the trigeminal vascular system, which 
transmits sensory information to the brain, so that the diencephalic nuclei could be affected during the aura phase [10] manifesting visual symptoms.

Perfusion by MRI shows the steady state of capillary blood flow that represents volume, speed, and oxygenation of the blood when it reaches a certain tissue that is measured intravascular, exogenous, and not diffusible with gadolinium [11]. In MA, some changes are reported during the aura or the onset of headache [4,6]; in our patient, however, the described findings of brain hypoperfusion during the headache phase suggest a generalized cerebral dysfunction, which could be due to spasm in the smaller arteries that suffer, and it may persist and influence the clinical manifestations during the headache phase. A state of generalized cerebral hyperperfusion has been suggested [4], and there may be a coexistence of both phenomena for some period.

A randomized, double-blind controlled study should be done in patients with similar characteristics. This may open a new line of research regarding the physiopathology and vascular changes of migraine in acute stages.

\section{Conclusion}

Cerebral hypoperfusion during the headache phase in migraine may be due to spasm of the capillary arteries. It may persist, conditioning the patients' symptoms during the headache phase. Also, a state of generalized brain hyperperfusion has been suggested, and there may be a coexistence of both phenomena for some period. More studies should be done in patients with similar characteristics. This may open a new line of research regarding the physiopathology and vascular changes of migraine in acute stages.

\section{Statement of Ethics}

The patient gave us a written consent. The study is exempt from ethics committee approval because this is a case report; because it is a case report with the written consent of the patient, it is not part of a research protocol. Written informed consent was obtained from the patient for publication of this case report and any accompanying images.

\section{Conflict of Interest Statement}

The authors declare that there are no additional disclosures to report.

\section{Funding Sources}

No specific funding was received for this work.

\section{Author Contributions}

J.L. contributed to conception, organization, execution, writing the first draft, review, and critique. J.C.B. contributed to organization, execution, writing the first draft, and review. C.G.-M. contributed to organization, design, execution, review, and critique. E.K. contributed to conception, design, review, and critique.

\section{Karger'}




\section{Data Availability Statement}

All data generated or analyzed during this study are included in this article. Further enquiries can be directed to the corresponding author.

\section{References}

1 Förster A, Wenz H, Kerl HU, Brockmann MA, Groden C. Perfusion patterns in migraine with aura. Cephalalgia. 2014 Oct;34(11):870-6.

2 Headache Classification Committee of the International Headache Society. The international classification of headache disorders. Cephalalgia. 2018 Jan;38(1):1-211.

3 Floery D, Vosko MR, Fellner FA, Fellner C, Ginthoer C, Gruber F, et al. Acute-onset migrainous aura mimicking acute stroke: MR perfusion imaging features. AJNR Am J Neuroradiol. 2012;33:1546-52.

4 Wolf ME, Okazaki S, Eisele P, Rossmanith C, Gregori J, Griebe M, et al. Arterial spin labeling cerebral perfusion magnetic resonance imaging in migraine aura: an Observational Study. J Stroke Cerebrovasc Dis. 2018 May; 27(5):1262-6.

5 Cobb-Pitstick KM, Munjal N, Safier R, Cummings DD, Zuccoli G. Time course of cerebral perfusion changes in children with migraine with aura mimicking stroke. AJNR Am J Neuroradiol. 2018 Sep;39(9):1751-5.

6 Blicher JU, Tietze A, Donahue MJ, Smith SA, Østergaard L. Perfusion and pH MRI in familial hemiplegic migraine with prolonged aura. Cephalalgia. 2016 Mar;36(3):279-83.

7 Moulton EA, Becerra L, Borsook D. An fMRI case report of photophobia: activation of the trigeminal nociceptive pathway. Pain. 2009 Oct;145(3):358-63.

8 Redon S, Donnet A. Sneezing in primary headaches with cranial autonomic symptoms: pathophysiological considerations. a series of case reports. Headache. 2018 Feb;58(2):298-303.

9 Buzzi MG, Formisano R, Colonnese C, Pierelli F. Chiari-associated exertional, cough, and sneeze headache responsive to medical therapy. Headache. 2003 Apr;43(4):404-6.

10 Akerman S, Holland PR, Goadsby PJ. Diencephalic and brainstem mechanisms in migraine. Nat Rev Neurosci. 2011 Sep;12(10):570-84.

11 Essig M, Nguyen TB, Shiroishi MS, Saake M, Provenzale JM, Enterline DS, et al. Perfusion MRI: the five most frequently asked clinical questions. AJR Am J Roentgenol. 2013 Sep;201(3):W495-510.

\section{Karger'א}

